OPEN ACCESS

Edited by:

Maria Schirone,

University of Teramo, Italy

Reviewed by:

Angelica Reyes-Jara,

University of Chile, Chile

Rolf Dieter Joerger,

University of Delaware, USA

*Correspondence:

Houhui Song

songhh@zafu.edu.cn

Weihuan Fang

whfang@zju.edu.cn

${ }^{\dagger}$ These authors have contributed equally to this work.

Specialty section:

This article was submitted to

Food Microbiology,

a section of the journal

Frontiers in Microbiology

Received: 06 December 2016 Accepted: 19 January 2017

Published: 31 January 2017

Citation:

Cheng C, Dong Z, Han X, Sun J,

Wang $H$, Jiang $L$, Yang $Y$, Ma $T$,

Chen $Z$, Yu J, Fang $W$ and Song $H$

(2017) Listeria monocytogenes

10403S Arginine Repressor ArgR

Finely Tunes Arginine Metabolism

Regulation under Acidic Conditions.

Front. Microbiol. 8:145.

doi: 10.3389/fmicb.2017.00145

\section{Listeria monocytogenes 104035 Arginine Repressor ArgR Finely Tunes Arginine Metabolism Regulation under Acidic Conditions}

\author{
Changyong Cheng ${ }^{1+}$, Zhimei Dong ${ }^{1+}$, Xiao Han ${ }^{1}$, Jing Sun ${ }^{1}$, Hang Wang ${ }^{1}$, Li Jiang', \\ Yongchun Yang ${ }^{1}$, Tiantian Ma ${ }^{1}$, Zhongwei Chen ${ }^{1}$, Jing Y ${ }^{1}{ }^{1}$, Weihuan Fang ${ }^{1,2 *}$ and \\ Houhui Song ${ }^{1 *}$
}

${ }^{1}$ College of Animal Science and Technology, China-Australia Joint-Laboratory for Animal Health Big Data Analytics, Zhejiang Provincial Engineering Laboratory for Animal Health Inspection \& Internet Technology, Zhejiang A\&F University, Lin'an, China, ${ }^{2}$ Zhejiang Provincial Key Laboratory of Preventive Veterinary Medicine, Institute of Preventive Veterinary Medicine, Zhejiang University, Hangzhou, China

Listeria monocytogenes is able to colonize human and animal intestinal tracts and to subsequently cross the intestinal barrier, causing systemic infection. For successful establishment of infection, $L$. monocytogenes must survive the low $\mathrm{pH}$ environment of the stomach. L. monocytogenes encodes a functional ArgR, a transcriptional regulator belonging to the ArgR/AhrC arginine repressor family. We aimed at clarifying the specific functions of ArgR in arginine metabolism regulation, and more importantly, in acid tolerance of $L$. monocytogenes. We showed that ArgR in the presence of $10 \mathrm{mM}$ arginine represses transcription and expression of the $\arg \mathrm{GH}$ and $\arg \mathrm{CJBDF}$ operons, indicating that $L$. monocytogenes ArgR plays the classical role of ArgR/AhrC family proteins in feedback inhibition of the arginine biosynthetic pathway. Notably, transcription and expression of $\operatorname{arc} A$ (encoding arginine deiminase) and sigB (encoding an alternative sigma factor B) were also markedly repressed by ArgR when bacteria were exposed to $\mathrm{pH} 5.5$ in the absence of arginine. However, addition of arginine enabled ArgR to derepress the transcription and expression of these two genes. Electrophoretic mobility shift assays showed that ArgR binds to the putative ARG boxes in the promoter regions of $\arg C, \arg G, \operatorname{arc} A$, and $\operatorname{sig} B$. Reporter gene analysis with gfp under control of the $\arg G$ promoter demonstrated that $A r g R$ was able to activate the $\arg G$ promoter. Unexpectedly, deletion of argR significantly increased bacterial survival in $\mathrm{BHI}$ medium adjusted to $\mathrm{pH} 3.5$ with lactic acid. We conclude that this phenomenon is due to activation of $\operatorname{arcA}$ and sigB. Collectively, our results show that $L$. monocytogenes ArgR finely tunes arginine metabolism through negative transcriptional regulation of the arginine biosynthetic operons and of the catabolic $\operatorname{arc} A$ gene in an arginine-independent manner during lactic acid-induced acid stress. ArgR also appears to activate catabolism as well as sigB transcription by anti-repression in an arginine-dependent way.

Keywords: Listeria monocytogenes, arginine repressor, ArgR, regulation, acid tolerance 


\section{INTRODUCTION}

Listeria monocytogenes is a foodborne bacterial pathogen capable of invasion and replication in phagocytic and nonphagocytic cells. This capacity allows it to cross protective epithelial barriers of the human body and cause severe infection with high mortality, especially in elderly populations, infants, immunocompromised individuals, and pregnant women (Cossart, 2011; Lebreton et al., 2015). The bacterium is resistant to acidic environments encountered during food processing, in acidic food, the stomach and phagosomes of macrophages (Cotter and Hill, 2003; Gray et al., 2006), and employs several mechanisms for $\mathrm{pH}$ homeostasis to survive or even proliferate in acidic conditions. L. monocytogenes utilizes the arginine deiminase (ADI) and agmatine deiminase (AgDI) systems to produce ammonia to neutralize intracellular protons by forming $\mathrm{NH}_{4}{ }^{+}$to elevate its cytoplasmic pH (Ryan et al., 2009; Chen et al., 2011; Cheng et al., 2013a,b). The general stress responsive alternative sigma factor sigma B (SigB or $\sigma \mathrm{B}$ ), which was first identified in Bacillus subtilis (Boylan et al., 1993), plays a pivotal role in response to environmental stresses in Listeria (Ferreira et al., 2001; Xia et al., 2016).

Arginine catabolism via the ADI pathway is widely distributed in bacteria enabling them to survive under harsh acidic environments and to evade host defenses (Marquis et al., 1987; Gruening et al., 2006; Lucas et al., 2007; Fulde et al., 2011; Xiong et al., 2015). The ADI pathway consists of three enzymes: ADI, ornithine carbamoyl-transferase and carbamate kinase, which are encoded by $\operatorname{arc} A, \operatorname{arcB}$ and $\operatorname{arc} C$, respectively. The actions of these three proteins convert arginine to ornithine, ammonia and carbon dioxide (Xiong et al., 2015). In many bacteria, the ADI pathway is regulated by an arginine repressor, $\mathrm{ArgR}$, a hexameric protein that belongs to the ArgR/AhrC family of transcriptional regulators involved in regulation of arginine biosynthetic metabolism in a feedback manner (Fulde et al., 2011; Choi et al., 2012; Xiong et al., 2015). Classical biosynthesis of arginine from glutamate is composed of eight enzymatic steps (Supplementary Figure S1A). Five steps involving $N$-acetylated intermediates contribute to formation of ornithine, and three additional steps are required to convert ornithine into arginine via several biosynthetic enzymes encoded by argABCDEFGH (Cunin et al., 1986). In the recycling pathway as In bacilli and most other prokaryotes, the acetyl group of $N$-acetylornithine is effectively transferred to glutamate by an acetyltransferase (ArgJ), making $N$-acetylglutamate synthase (ArgA), and $N$-acetylornithinase (ArgE) of the linear pathway redundant (Lu, 2006). This situation is also found in L. monocytogenes (Supplementary Figure S1B). Generally, ArgR proteins regulate their target genes by binding to the operator sites (called ARG box), leading to repression of arginine biosynthetic genes and activation of catabolic genes in the presence of arginine. ArgR proteins also regulate various genes involved in arginine transport (Maghnouj et al., 1998; Caldara et al., 2006). ArgR-mediated regulation network has been shown to respond to various environmental stimuli, such as changes in concentration of arginine and other metabolites and fluctuations in $\mathrm{pH}$, temperature, and oxygen tension (Dong et al., 2004; Gruening et al., 2006; Xiong et al., 2015).
Homologs of the ADI and the arginine biosynthesis pathway genes have been found in the sequenced genome of L. monocytogenes strain EGD-e by in silico analysis (Glaser et al., 2001). The ADI encoded by $\operatorname{arcA}$, is a critical enzyme in the ADI system that triggers the first reaction. The molecular characteristics of the ADI system and its contributions to acid tolerance of L. monocytogenes in vitro have been studied (Ryan et al., 2009; Cheng et al., 2013b), but the underlying regulatory mechanisms have not been determined. Moreover, SigB is an important component that links survival to environmental stress and virulence in L. monocytogenes and is involved in the regulation of more than 150 genes (Palmer et al., 2011; Xia et al., 2016). Nevertheless, little is known about the regulation of transcription and expression of SigB in L. monocytogenes. Here, we report that L. monocytogenes ArgR is a negative regulator of the expression, not only of arginine biosynthesis genes ( $\operatorname{argCJBD}$ and $\arg G H)$ but also of ArcA, essential for arginine catabolism, and of SigB. Such regulation might occur by direct interaction with its ARG boxes in the promoter regions. Most notably, we determined that ArgR plays a unique role in acidic tolerance of L. monocytogenes by exerting a regulatory role on $\operatorname{arc} A$ and $\operatorname{sig} B$.

\section{MATERIALS AND METHODS}

\section{Bacterial Strains, Plasmids, and Culture Conditions}

Listeria monocytogenes 10403 S was used as the wild-type strain. Escherichia coli $\mathrm{DH} 5 \alpha$ was employed for cloning experiments and as the host strain for plasmids pET30a(+; Merck, Darmstadt, Germany), pERL3 and pKSV7. E. coli Rosetta (DE3) was used for prokaryotic protein expression. L. monocytogenes was cultured in brain heart infusion (BHI) medium (Oxoid, Hampshire, England). DH5 $\alpha$ and Rosetta (DE3) cells were grown at $37^{\circ} \mathrm{C}$ in Luria-Bertani broth (LB; Oxoid). Stock solutions of ampicillin $(50 \mathrm{mg} / \mathrm{ml})$, erythromycin $(50 \mathrm{mg} / \mathrm{ml})$, kanamycin $(50 \mathrm{mg} / \mathrm{ml})$, or chloramphenicol $(50 \mathrm{mg} / \mathrm{ml})$ were added to media when necessary. All chemicals were obtained from Sangon Biotech (Shanghai, China), Merck or Sigma-Aldrich (St. Louis, MO, USA) and were of the highest available purity.

\section{Bioinformatics Analysis}

Alignment of nucleotide and deduced amino acid sequences was performed with MUSCLE by using Geneious software (Edgar, 2004). The amino acid sequences of ArgR of L. monocytogenes 10403S strain and its homologs in other microbial species were obtained from the National Centre for Biotechnology Information database (NCBI). The known crystal structure of B. subtilis ArgR (PDB: 1F9N) was acquired from the Protein Data Bank (PDB). A putative model of L. monocytogenes ArgR was constructed using SWISS-MODEL Workspace (Arnold et al., 2006; Bordoli et al., 2009; Bordoli and Schwede, 2012). Promoters of genes of interest from the L. monocytogenes 10403S genome sequence were identified using the BPROM modules of the Softberry website $^{1}$. This program gives output scores from

\footnotetext{
${ }^{1}$ http://www.softberry.com/
} 
-1 to $\sim 25$ to estimate the likelihood that a predicted promoter is functional and a higher score indicates that the prediction is more likely to be correct. ArgR binding sites composed of two palindrome sequences, known as ARG boxes, have been identified previously in several bacteria species (Larsen et al., 2005; Kloosterman and Kuipers, 2011; Perez-Redondo et al., 2012). Promoter/operator elements containing ARG box motifs were identified by searching the L. monocytogenes genome with a position weight matrix derived from known E. coli ArgR recognition elements (Chen et al., 1997), using the Virtual Footprint software program ${ }^{2}$ (Munch et al., 2005).

\section{Construction of Gene Deletion Mutant}

The temperature-sensitive pKSV7 shuttle vector was used for generating mutations in L. monocytogenes 10403S. A homologous recombination strategy with the splicing by overlap extension (SOE) PCR procedure was used for in-frame deletion to construct gene deletion mutants (Monk et al., 2008). DNA fragments containing homologous arms upstream and downstream of the gene of interest were obtained via amplification of $10403 \mathrm{~S}$ genomic DNA using the primer pairs listed in Supplementary Table S1. The obtained fragment was then cloned into pKSV7 and transformed into $\mathrm{DH} 5 \alpha$. After confirmation by sequencing, the recombinant vector containing the target gene deletion cassette was electroporated into the competent L. monocytogenes cells. Transformants were selected on BHI agar plates containing chloramphenicol $(10 \mu \mathrm{g} / \mathrm{ml})$. A single transformant was serially passaged at a non-permissive temperature $\left(41^{\circ} \mathrm{C}\right)$ in $\mathrm{BHI}$ medium containing chloramphenicol to promote chromosomal integration. A single colony with chromosomal integration was successively passaged in $\mathrm{BHI}$ medium without chloramphenicol at a permissive temperature $\left(30^{\circ} \mathrm{C}\right)$ to enable plasmid excision and curing (Camilli et al., 1993). Recombinants were identified as chloramphenicol-sensitive colonies, and mutagenesis was further confirmed by PCR and DNA sequencing. The single mutant strain was used in a second round of mutagenesis to construct double deletion mutants.

\section{Complementation of $\arg R$ Deletion Mutant}

To complement the L. monocytogenes $\Delta$ ArgR strain, the complete $\operatorname{argR}$ open reading frame (ORF) along with its promoter was amplified from genomic DNA using primer pairs listed in Supplementary Table S1. After digestion with appropriate enzymes, the PCR product was cloned into pERL3, a plasmid capable of replication in Gram positive bacteria. The resulting plasmid was then electroporated into the L. monocytogenes $\triangle \mathrm{ArgR}$ strain. Plasmid-containing cells were selected on BHI agar plates containing erythromycin $(10 \mu \mathrm{g} / \mathrm{ml})$. The complemented strain was designated as $\mathrm{C} \Delta \mathrm{ArgR}$.

\section{Expression and Purification of Recombinant Proteins}

The recombinant proteins used in this study were expressed as fusion proteins to the N-terminal His-tag using pET30a $(+)$ as

${ }^{2}$ http://prodoric.tu-bs.de/ the expression vector. Rosetta (DE3) was used as the expression host. The full-length ORF of the gene of interest from the 10403S genome was amplified with the primer pair listed in Supplementary Table S1 and inserted into the pET30a $(+)$ vector, and finally transformed into Rosetta competent cells. E. coli cells harboring recombinant plasmids were grown in $250 \mathrm{~mL}$ LB supplemented with $50 \mu \mathrm{g} / \mathrm{mL}$ kanamycin at $37^{\circ} \mathrm{C}$ until cultures reached $1.2-1.4$ at $\mathrm{OD}_{600} \mathrm{~nm}$. Isopropyl $\beta-\mathrm{D}-1-$ thiogalactopyranoside (IPTG) was added to a final concentration of $0.4 \mathrm{mM}$ to induce expression of interest proteins for an additional $3-4 \mathrm{~h}$ at $30^{\circ} \mathrm{C}$ in the form of soluble protein. His-tagged fusion proteins were purified using nickel-chelated affinity column chromatography (Weishi-Bohui Chromtotech Co., Beijing, China). Specifically, IPTG-induced cell pellets were collected, re-suspended in $50 \mathrm{mM}$ PBS ( $\mathrm{pH}$ 7.4) and disrupted by sonication. After centrifugation at 12,000 $\mathrm{g}$ for $30 \mathrm{~min}$, the soluble protein samples were collected and loaded onto a $1 \mathrm{ml}$ pre-packed nickel-chelated agarose gel column according to the manufacturer's instructions. Finally, expression and purification of recombinant proteins were analyzed via $10 \%$ SDS-PAGE followed by Coomassie brilliant blue staining and protein concentration was quantified with the Bradford method.

\section{Preparation of Polyclonal Antibodies against Recombinant Proteins}

Rabbits were initially immunized via subcutaneous injection of $500 \mu \mathrm{g}$ protein with an equal volume of Freund's complete adjuvant (Sigma). After 2 weeks, rabbits were given subcutaneous booster injections of $250 \mu \mathrm{g}$ protein each in incomplete Freund's adjuvant (Sigma) three times at 10 -day intervals. Rabbits were bled $\sim 10$ days after the last injection.

\section{Producing a Truncated ArgR by Site-Directed Mutagenesis}

To identify the predicted active sites of ArgR, a double mutant (S42AR43A) was generated using the original vector template, pET30a-ArgR, and the QuikChange Site-Directed Mutagenesis kit (Agilent, Santa Clara, CA, USA) with the primer pairs described in Supplementary Table S1. Template DNA was removed via digestion with $D p n \mathrm{I}$ (TOYOBO, Osaka, Japan) for $2 \mathrm{~h}$ at $37^{\circ} \mathrm{C}$. The mutant construct was sequenced to ensure that only the desired single mutations had been incorporated correctly. The corresponding mutant protein was designated $\operatorname{ArgR}_{S 42 \mathrm{AR} 43 \mathrm{~A}}$, and expressed and purified as described above.

\section{Crosslinking Analysis}

The purified N-terminal 6-histidine-tagged ArgR proteins were crosslinked with various amounts of glutaraldehyde (Sigma) in $50 \mathrm{mM}$ HEPES ( $\mathrm{pH} \mathrm{8.0)}$ ), containing $150 \mathrm{mM} \mathrm{KCl}$ and $1 \mathrm{mM}$ $\mathrm{L}$-arginine. The reaction mixture was incubated with or without $1 \% \beta$-mercaptoethanol at room temperature for $2 \mathrm{~h}$ and the crosslinked ArgR complexes were analyzed by $10 \%$ SDS-PAGE, and stained with Coomassie Brilliant Blue. 


\section{Electrophoretic Mobility Shift Assay (EMSA)}

DNA binding of $\operatorname{ArgR}$ and its mutant $\operatorname{ArgR}_{\text {S42AR43A }}$ was investigated in vitro by using electrophoretic mobility shift assay (EMSA). The DNA fragment of the promoter region of $\arg C, \arg G, \operatorname{arcA}$, or $\operatorname{sig} B$ containing the putative ARG box was generated by PCR with the specific primer pairs (Supplementary Table S1). The DNA fragments were purified with a PCR Purification Kit (Sangon). Then 200 ng DNA was incubated with varying concentrations of purified recombinant $\operatorname{ArgR}$ or $\operatorname{ArgR}_{S 42 \mathrm{AR} 43 \mathrm{~A}}$ in binding buffer $(50 \mathrm{mM}$ Tris- $\mathrm{HCl}, \mathrm{pH}$ 8.0, $250 \mathrm{mM} \mathrm{NaCl}, 5.0 \mathrm{mM} \mathrm{MgCl}_{2}$, $2.5 \mathrm{mM}$ DTT, $2.5 \mathrm{mM}$ EDTA, and $20 \%$ glycerol) for $30 \mathrm{~min}$ at room temperature. ProteinDNA complexes were separated electrophoretically on a native $5 \%$ polyacrylamide gel at $80 \mathrm{~V}$ with $0.5 \mathrm{x}$ Tris-acetate-EDTA (TAE) buffer and visualized using ethidium bromide staining.

\section{Construction of $\mathrm{P}_{\text {arg }}$ Fusing gfp Reporter Strains and Promoter Studies}

For transcriptional fusion of the $\arg G$ promoter $\left(\mathrm{P}_{\arg G}\right)$ to the GFP reporter protein, the fragment containing the promoteroperator region of the $\operatorname{argGH}$ operon was amplified with the primer pair listed in Supplementary Table S1 using genomic DNA from L. monocytogenes $10403 \mathrm{~S}$ as template. In parallel, the promoterless $g f p$ allele $g f p$ mut $3^{*}$ was amplified from the Listeria shuttle vector pAMGFP3 using primers listed in Supplementary Table S1. The two fragments were fused by using overlapping PCR. The resulting fragment containing the promoter- $g f p$ fusion was cloned into vector pERL3 to generate the reporter plasmid which was then electroporated into the wildtype $10403 \mathrm{~S}$ or the $\Delta \mathrm{ArgR}$ strain. Transformants were selected by plating onto erythromycin-containing BHI agar plates. For promoter studies, L. monocytogenes was grown to stationary phase $\left(\mathrm{OD}_{600 \mathrm{~nm}}=1.2\right)$ in $\mathrm{BHI}$ broth at $37^{\circ} \mathrm{C}$, and then exposed to acidic ( $\mathrm{pH}$ 5.5) or neutral ( $\mathrm{pH}$ 7.0) conditions for an additional $60 \mathrm{~min}$. Bacteria in $1 \mathrm{~mL}$ of culture were harvested by centrifugation, the cell pellets were washed once with $10 \mathrm{mM}$ PBS ( $\mathrm{pH}$ 7.4) and resuspended in $1 \mathrm{~mL}$ of $10 \mathrm{mM}$ PBS. One hundred microliters of the suspension was used for $g f p$ measurements and fluorescence observation. For the former, relative fluorescence unites (RFU) were measured in a fluorescence reader (BioTek Synergy H1, Winooski, VT, USA) with excitation at $485 \mathrm{~nm}$ and emission at $535 \mathrm{~nm}$. Relative fluorescence values were calculated by subtracting extinction from the PBS background. For the latter, fluorescence intensity was observed by using confocal laser scanning microscopy (FLV 1000; Olympus, Japan).

\section{Survival in Acidic Conditions}

Cells from stationary phase cultures of L. monocytogenes 10403S, mutants ( $\triangle \operatorname{ArgR}, \Delta \operatorname{SigB}$, and $\Delta \operatorname{ArgR} \Delta \operatorname{SigB}$ ) and complemented strain $\mathrm{C} \triangle \mathrm{ArgR}$ were harvested, washed in PBS and re-suspended in BHI (adjusted to $\mathrm{pH} 3.5$ with $3 \mathrm{M}$ lactic acid). After 30, 60, $90,120,160$, or $200 \mathrm{~min}$ of incubation at $37^{\circ} \mathrm{C}$, the surviving cells were plated onto BHI agar after appropriate dilutions. The plates were incubated at $37^{\circ} \mathrm{C}$ for $24 \mathrm{~h}$ and survival rates are reported as the mean of three independent experiments, which were performed in duplicate.

\section{Real-Time Quantitative RT-PCR (qRT-PCR)}

Listeria monocytogenes wild-type $10403 \mathrm{~S}$ and its mutant strain $\triangle \mathrm{ArgR}$ were grown to the stationary phase $\left(\mathrm{OD}_{600 \mathrm{~nm}}=1.2\right)$ in $\mathrm{BHI}$ broth at $37^{\circ} \mathrm{C}$, and then exposed to acidic ( $\left.\mathrm{pH} 5.5\right)$ and neutral $(\mathrm{pH}$ 7.0) conditions, respectively, for additional $1 \mathrm{~h}$. Total RNA was extracted using the Column Bacterial total RNA Purification Kit (Sangon), according to the manufacturer's instructions, genomic DNA removed using DNase I (TaKara, Japan) and cDNA synthesized with reverse transcriptase (TOYOBO, Osaka, Japan). Real-time quantitative PCR was performed in a $20 \mu \mathrm{L}$ reaction volume containing $200 \mathrm{ng}$ cDNA, $10 \mu \mathrm{L}$ SYBR quantitative PCR mix (TOYOBO), and $1 \mu \mathrm{L}$ genespecific primers (200 nM, Supplementary Table S1) to measure the transcriptional levels of $\operatorname{arc} A, \operatorname{sig} B, \arg C$, and $\arg G$ using the Mx3000P PCR detection system (Agilent). The housekeeping gene, $g y r B$, was used as an internal control for normalization in each sample as previously described (Chen et al., 2011). Relative transcription levels were quantified using the $2^{-\Delta \Delta C T}$ method and shown as relative fold changes (Livak and Schmittgen, 2001). Triplicate assays were performed for each gene.

\section{Preparation of Whole-Cell Lysates and Western Blot Analysis}

Bacteria were grown in BHI broth to the stationary growth phase and lysates were prepared as described before (Ryan et al., 2009). Specifically, the stationary bacteria exposed to acidic ( $\mathrm{pH}$ 5.5) and neutral ( $\mathrm{pH} 7.0)$ conditions, respectively, for additional $1 \mathrm{~h}$. Bacterial pellets were then re-suspended in $1 \mathrm{~mL}$ of extraction solution (2\% Triton X-100, 1\% SDS, $100 \mathrm{mM} \mathrm{NaCl}, 10 \mathrm{mM}$ Tris$\mathrm{HCl}, 1 \mathrm{mM}$ EDTA, $\mathrm{pH}$ 8.0). One gram of glass beads (G8772, Sigma) was added and samples lysed using the homogenizer Precellys 24 (Bertin, Provence, France) at $6000 \mathrm{rpm}$ for $30 \mathrm{~s}$ with intermittent cooling for $30 \mathrm{~s}$ (two cycles in total), followed by centrifugation at 12,000 g for $15 \mathrm{~min}$. Supernatants were retained as cell-free extracts. Samples containing equal amounts of protein were subjected to $12 \%$ SDS-PAGE and the separated proteins were blotted onto $0.22 \mu \mathrm{m}$ polyvinylidene difluoride (PVDF) membranes (Merck Millipore). Membranes were blocked for $1 \mathrm{~h}$ with $5 \%$ skimmed milk, and incubated for $1 \mathrm{~h}$ with polyclonal antisera against recombinant recombinant ArgR, ArgG, SigB, or ArcA in $0.5 \%$ skimmed milk. Next, membranes probed with antiinterest protein were developed using HRP-conjugated goat antirabbit IgG (Santa Cruz, California, CA, USA) as the secondary antibody. Chemiluminescence was detected via a bio-imaging system (UVP EC3 Imaging System, UVP Inc., Upland, CA, USA), and the densities of the interest protein bands were normalized to the GAPDH signal and quantified using Quantity One software (Bio-Rad).

\section{Statistical Analysis}

All data comparisons were analyzed using the two-tailed Student $t$-test. Differences with $P$-values of 0.05 were considered 


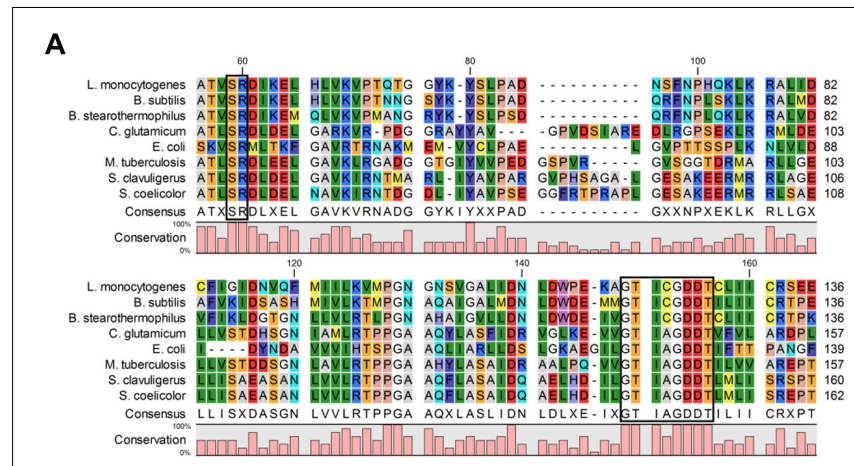

C

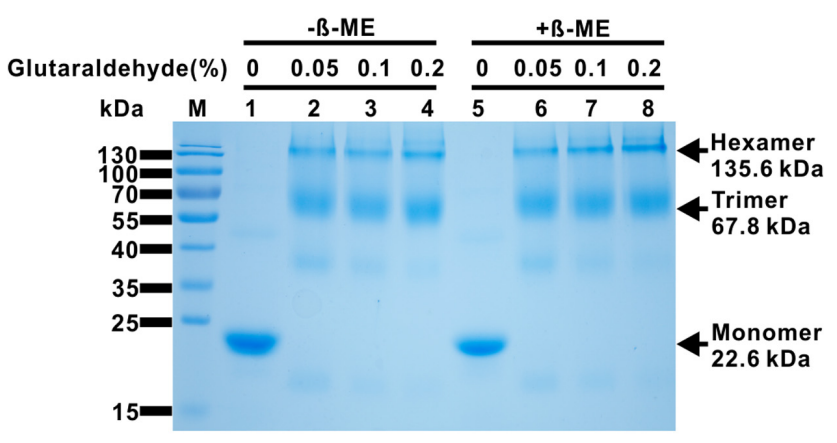

B

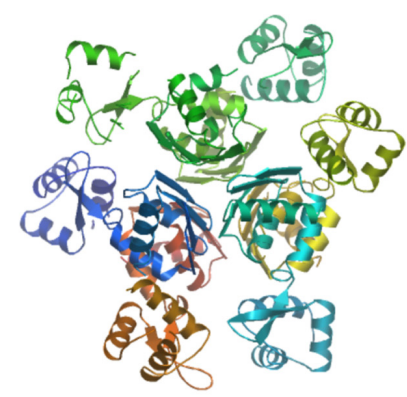

B. subtilis ArgR (PDB: 1F9N)

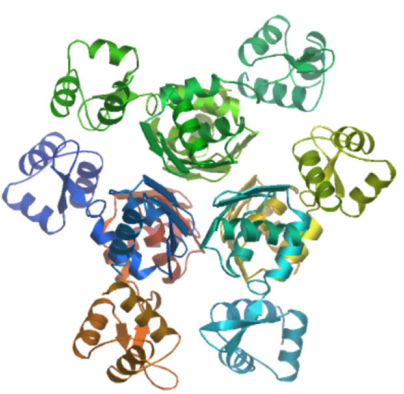

L. monocytogenes ArgR
D

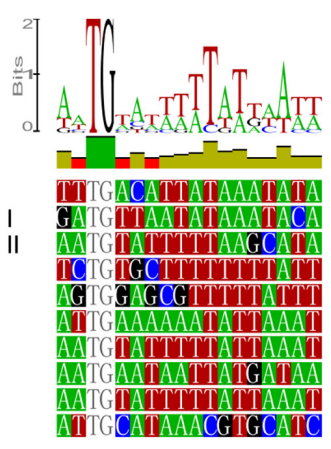

FIGURE 1 | Listeria monocytogenes ArgR protein is a member of ArgR/AhrC family transcriptional regulators. (A) Amino acid sequence alignment of L. monocytogenes ArgR against the members of the ArgR/AhrC family from Bacillus subtilis, B. stearothermophilus, Corynebacterium glutamicum, Escherichia coli, Mycobacterium tuberculosis, Streptomyces clavuligerus, and S. coelicolor. The two conserved motifs that are responsible for DNA and arginine binding are blackened. (B) Predicted tertiary fold of $L$. monocytogenes ArgR using the B. subtilis ArgR (PDB: 1F9N) as the template in the SWISS-MODEL Workspace. (C) SDS-PAGE analysis of glutaraldehyde crosslinking of $L$. monocytogenes ArgR for the identification of protein polymers. The monomeric, trimeric and hexameric proteins are indicated by arrows. (D) Promoter/operator elements containing binding sites of ArgR (ARG box) were identified by searching the $L$. monocytogenes genome with a position weight matrix derived from known ArgR recognition elements using the Virtual Footprint software program (as described in detail in the Materials and Methods). The identified potential consensus binding sites of ArgR in the promoter region from $L$. monocytogenes gene $\arg C, \arg G$, $\operatorname{arc} A$, and $\operatorname{sig} B$ were further aligned with those from B. subtilis, B. licheniformis, and S. coelicolor.

statistically significant, and those with $P$-values of 0.01 were considered markedly statistically significant.

\section{RESULTS}

\section{L. monocytogenes ArgR Is Predicted as a Typical Arginine Repressor}

The best characterized ArgR homolog from Gram-positive bacteria is AhrC from B. subtilis, as its crystal structure was determined in 2002 (PDB ID: 1F9N; Garnett et al., 2007). To better characterize L. monocytogenes ArgR, the amino acid sequence of this protein was aligned with those from seven other bacterial species. The alignment showed sequence identities ranging from 22 to $65 \%$, the highest percentage was exhibited between ArgR from L. monocytogenes and AhrC from B. subtilis (Figure 1A). Based on the amino acid sequence analysis, the L. monocytogenes ArgR monomer appears to possess two highly conserved motifs, the "SR" motif for DNA binding (residues 42-43) in the N-terminal region, and the "GTICGDDT" motif for arginine binding (residues 120-127) and oligomerization (residues 125-126) located in the C-terminal region (Figure 1A).
Furthermore, we modeled L. monocytogenes ArgR in the SWISSMODEL Workspace using the crystal structure of $B$. subtilis AhrC as the template. The predicted structure L. monocytogenes ArgR is of high similarity to that of AhrC. Specifically, the monomer ArgR also associates via its C-terminal (core) domain to form a hexamer, probably as a result of the face-to-face association of a pair of trimers (Dennis et al., 2002). The six C-terminal domains strictly follow the 32 non-crystallographic symmetry (NCS) and domains from each trimer locate above another one, giving the hexameric core a stacked configuration when viewed along the threefold axis. The DNA-binding domains (DBD) adopt slightly different positions around the periphery of the core and deviate from strict NCS (Figure 1B). The recombinant $\operatorname{ArgR}$ protein was expressed in E. coli, purified and subjected to crosslinking analysis using glutaraldehyde. The his-tagged ArgR protein had a molecular weight of about $23 \mathrm{kDa}$, and was able to form higher order multimeric complexes (mainly formed as the trimers and hexamers) in the presence of $0.05 \%$ glutaraldehyde (Figure 1C). These results suggest that ArgR of L. monocytogenes is a typical arginine repressor which might contribute to transcriptional regulation of arginine metabolism. 
A

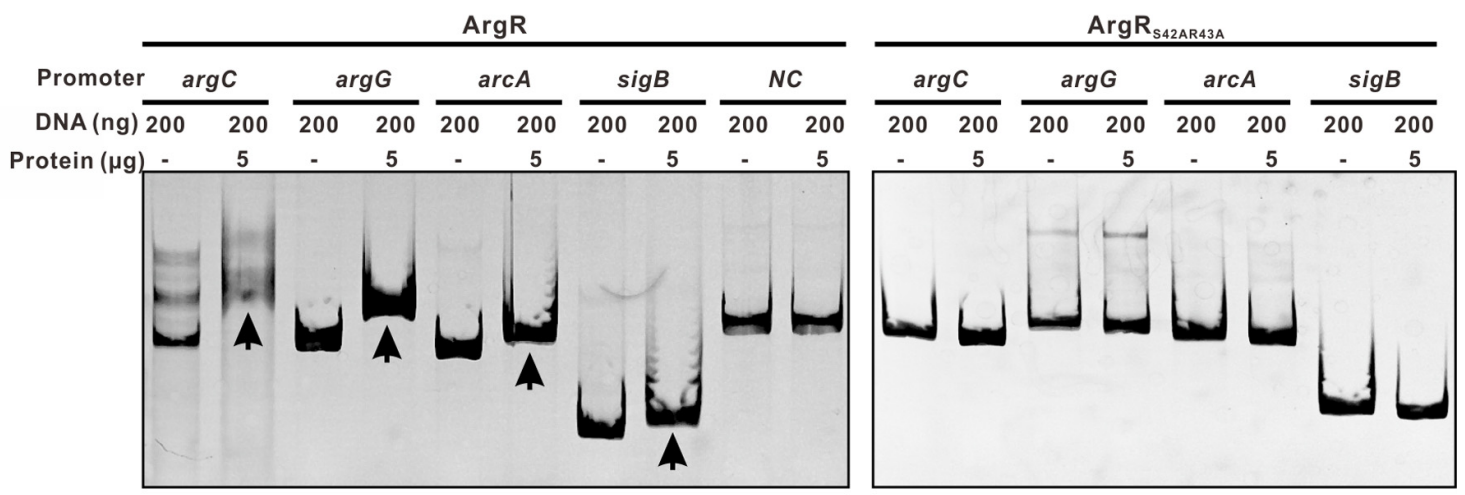

B

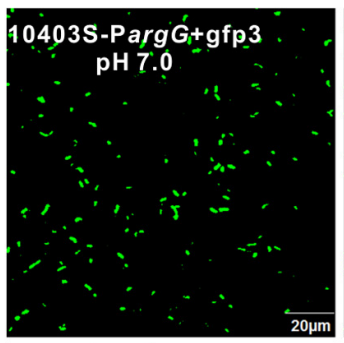

10403S-PargG+gfp3 pH 5.5

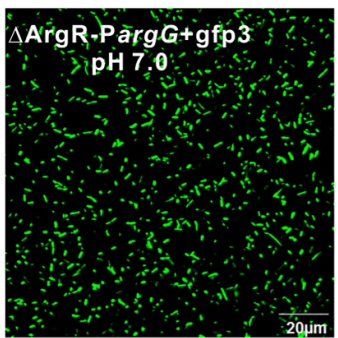

$\triangle A$ ArgR-Ṕarǵg $+g$ fp 3

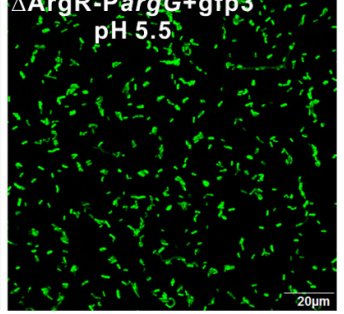

C

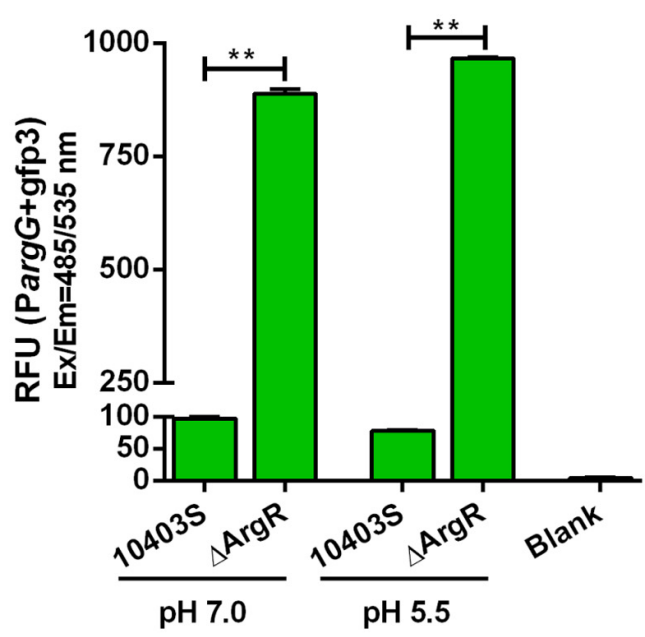

FIGURE 2 | (A) ArgR binds in vitro to the operating sites of $\arg C$, argG, arcA and sigB, and activates the argG operon promoter. Gel mobility shift assay (EMSA)

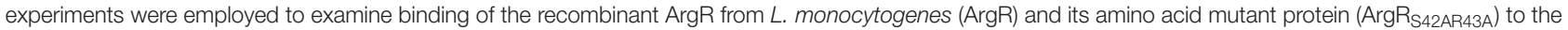
$\arg$, argG, arcA, and sigB promoter region DNA. The promoter fragments were obtained by PCR with primers specified in Supplementary Table S1, and incubated with recombinant proteins for $30 \mathrm{~min}$ at room temperature. Gel retardation by DNA-protein complexes was monitored after ethidium bromide staining. The housekeeping gene gyrB was used as a negative control (NC) for the EMSAs. Arrows indicate DNA-protein complexes. (B,C) ArgR activates the argG operon promoter. The fluorescence intensity was observed by confocal laser scanning microscopy of overnight grown $L$. monocytogenes wild-type and ArgR mutant strains carrying a gfp3 reporter fused with the promoter of arg G, and then stress-treated for an additional $1 \mathrm{~h}$ under $\mathrm{pH} 7.0$ and $5.5 \mathrm{conditions}$ (B). Bars represent the relative fluorescence units (RFU) after subtracting the absolute values for the PBS control (C). Data shown represents the Mean \pm SD of three independent experiments, each performed in duplicate. ${ }^{* *} P<0.01$ for comparisons between the wild-type and mutant strains.

\section{ARG Boxes are Present in the Promoter Regions of $\arg C J B D$, argGH, sigB, and $\operatorname{arcA}$}

We further analyzed the sequences of the promoter regions of $\operatorname{argCJBD}, \arg G H, \operatorname{sig} B$, and $\operatorname{arcA}$ genes for possible ArgR binding sites using a virtual footprint promoter analysis program (see Materials and Methods; Munch et al., 2005). The ARG box consensus was described as TNTGAATWWWWATTCANW in E. coli (Maas, 1994), CATGAATAAAAATKCAAK in B. subtilis (Miller et al., 1997), and AWTGCATRWWYATGCAWT in Streptomyces (Rodriguez-Garcia et al., 1997; where $\mathrm{W}=\mathrm{A}$ or $\mathrm{T}, \mathrm{K}=\mathrm{G}$ or $\mathrm{T}, \mathrm{R}=\mathrm{A}$ or $\mathrm{G}, \mathrm{Y}=\mathrm{T}$ or $\mathrm{C}, \mathrm{N}=$ any base). Five putative ARG boxes were identified in each putative promoter region upstream of these five genes from L. monocytogenes on the basis of similarity with the B. subtilis (Makarova et al., 2001) and B. licheniformis (Maghnouj et al., 1998) and there were 1-3 bp mismatch with respect to the consensus sequence (Figure 1D).

\section{ArgR Binds In vitro to the ARG Boxes of $\arg C J B D$, $\arg G H, \operatorname{sig} B$, and $\operatorname{arc} A$ Promoters}

To confirm that ArgR directly binds to the respective ARG boxes identified above, the EMSA was performed. The promoter regions containing the putative $A R G$ boxes were generated and incubated with recombinant ArgR, and the protein-DNA complexes were assayed by native gel electrophoresis. Figure 2A shows that recombinant $\mathrm{ArgR}$ was able to bind to DNA oligonucleotides of each promoter of $\operatorname{argCJBD}, \arg G H$, sigB, or 


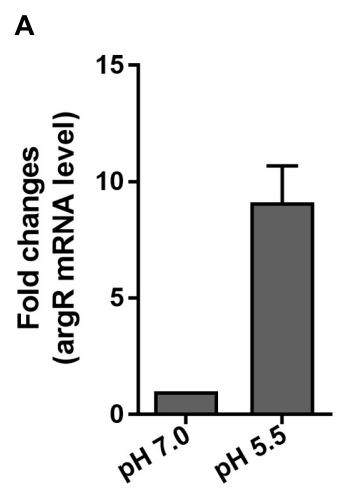

D $10 \mathrm{mM}$ arginine

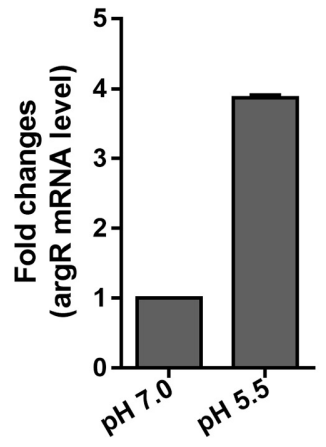

B

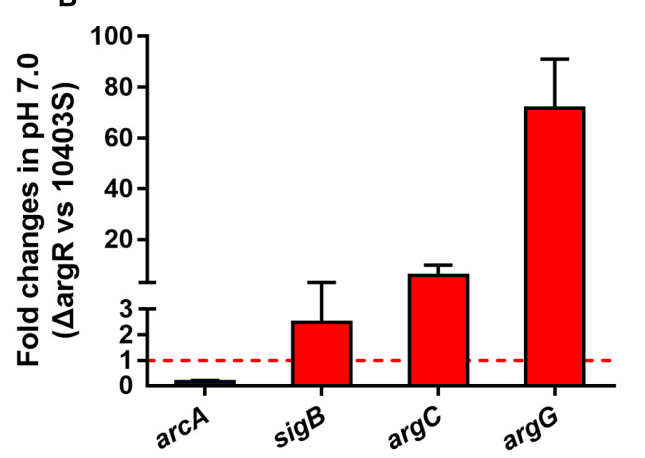

E

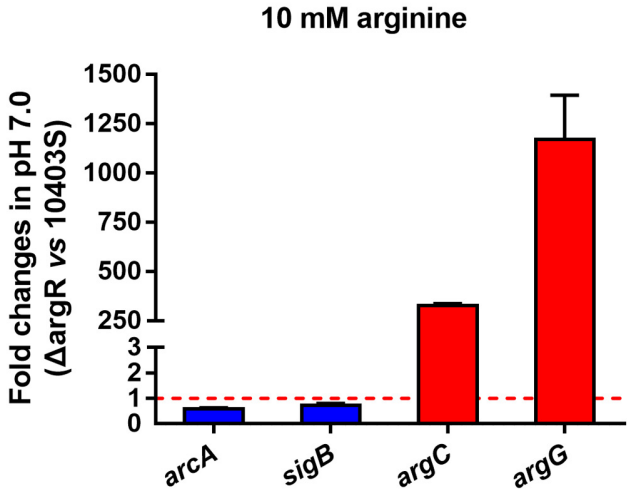

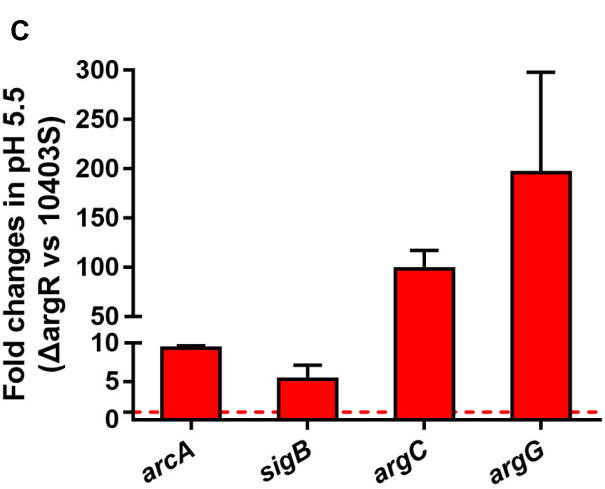

$\mathbf{F}$

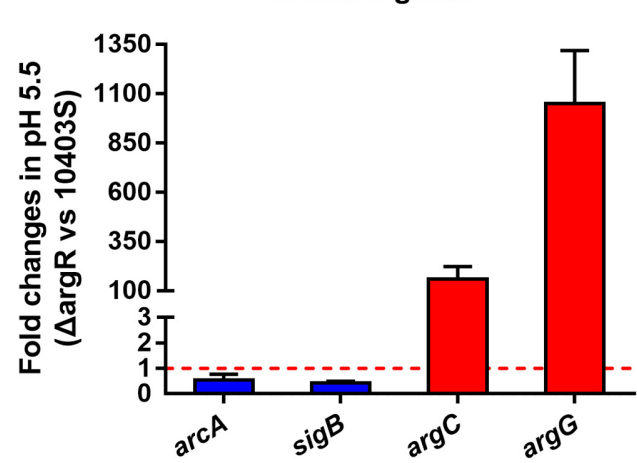

FIGURE 3 | ArgR regulates the transcription of $\arg C, \arg G, \operatorname{arcA}$, and sigB using arginine as a cofactor. Relative quantification of $\arg R, \operatorname{arc} A, \operatorname{sig} B$, $\arg C$, and $\arg G \mathrm{mRNA}$ and protein expression levels in L. monocytogenes wild-type and $\triangle \mathrm{ArgR}$ mutant strains under different pH conditions (7.0 and 5.5) in the presence (A-C) or absence (D-F) of exogenous arginine (10 mM). Values are expressed as Mean \pm SD. The dotted lines indicate the onefold change in transcription of the interest genes.

$\operatorname{arcA}$. More significantly, ArgR shows stronger binding capacity to $\operatorname{argCJBD}$ and $\arg G H$ promoters than to those of $\operatorname{sig} B$ and $\operatorname{arcA}$ under the experimental conditions we studied. These results indicate that $L$. monocytogenes ArgR contributes to overall regulation of the $\operatorname{argCJBD}, \arg G H, \operatorname{sig} B$, and $\operatorname{arc} A$ promoter activities although the degree of regulation could be different. More importantly, we found that ArgR protein completely lost the binding ability to ARG boxes when the two residues Ser42 and $\operatorname{Arg} 43$ (SR motif) were mutated to alanine (Figure 2A), strongly suggesting that these two sites are critical amino acids for ArgR-DNA binding.

\section{ArgR Activates the argG Operon Promoter}

To further analyze the regulatory function of $\mathrm{ArgR}$ in expression of its target genes, we cloned a DNA fragment covering the promoter-operator region of the $\arg G H$ operon (as the representative gene cluster involved in arginine anabolism) into the $g f p$ reporter vector which was transformed into $\triangle \mathrm{ArgR}$ mutant and its parent strain. Data show that GFP expression was significantly elevated in the $\triangle \mathrm{ArgR}$ mutant under neutral and acidic conditions while GFP was barely detectable in the wildtype strain (Figures 2B,C). Thus, ArgR is shown to repress the arginine biosynthetic pathway by interacting with the promoter of the $\arg G H$ operon.

\section{ArgR Regulates Transcription and Expression of $\arg C, \arg G, \operatorname{arc} A$, and $\operatorname{sig} B$}

Since the putative ARG boxes are present in the promoter regions of $\arg C, \arg G, \operatorname{arc} A$, and $\operatorname{sig} B$, transcription of these genes might be regulated by ArgR. To find out if such regulation occurs, real-time quantitative PCR was performed using total RNA isolated from the wild-type strain L. monocytogenes $10403 \mathrm{~S}$ and the $\arg R$ deletion mutant $\triangle \mathrm{ArgR}$ in the presence $(10 \mathrm{mM})$ or absence of arginine. We found that expression of $\operatorname{argR}$ was significantly induced in response to acidic $\mathrm{pH}$ at 5.5 regardless of arginine supplementation (Figures 3A,D). The transcriptional levels of two representative genes ( $\arg C$ and $\arg G$ ) involved in arginine anabolism were significantly increased in the $\triangle \mathrm{ArgR}$ mutant under neutral or acidic conditions (Figures 3B,C), and such effects were augmented by addition of exogenous arginine (Figures 3E,F). These findings indicate that L. monocytogenes ArgR plays a classical role of ArgR/AhrC family in feedback inhibition of the arginine biosynthetic pathway using arginine as a corepressor. Transcription of $\operatorname{arcA}$ was downregulated in the $\triangle \mathrm{ArgR}$ mutant under neutral $\mathrm{pH}$ (Figure 3B), which is consistent with findings from the previous study by Ryan et al. (2009), 


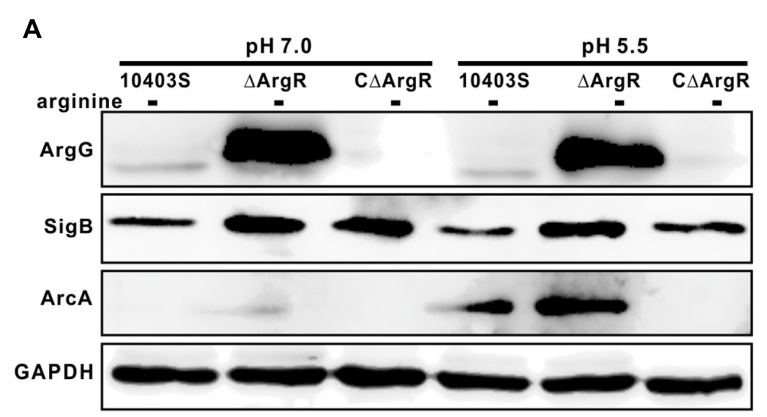

B
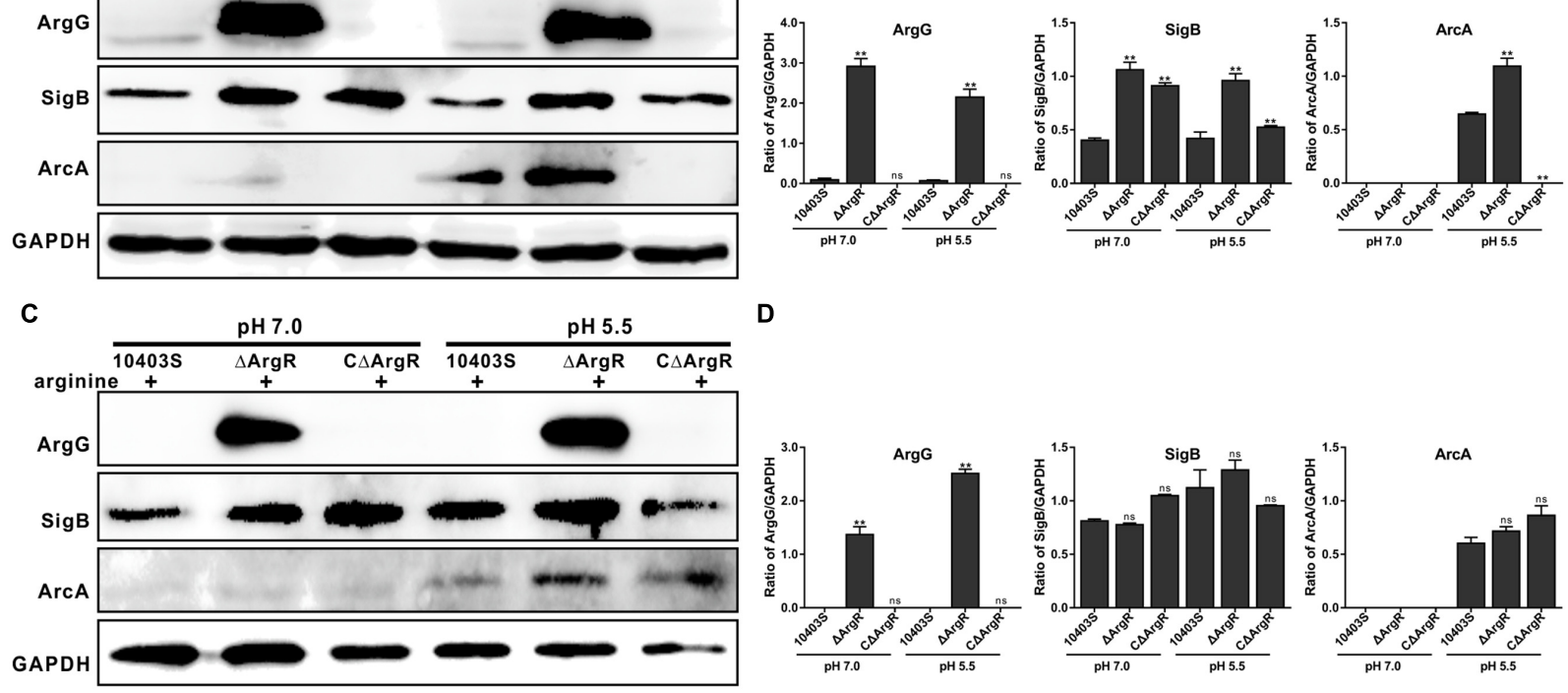

D
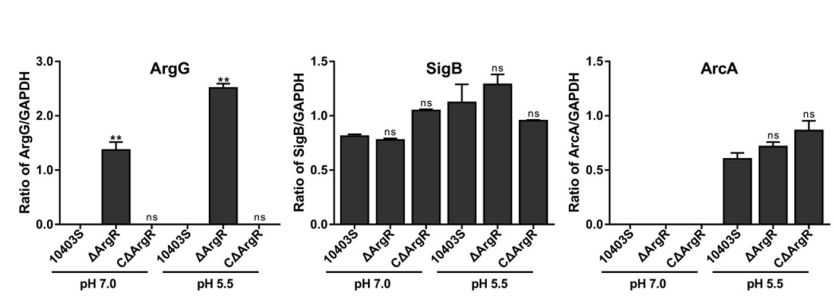

FIGURE 4 | ArgR regulates the expression of ArgG, SigB, and ArcA using arginine as a cofactor. Total bacterial cell-free protein was isolated $2 \mathrm{~h}$ after stress under different pH conditions (7.0 and 5.5) in the absence (A) or presence (C) of exogenous arginine (10 mM), and the protein expression levels of ArgG, SigB, and ArcA were then determined by Western blotting. GAPDH was used as an internal control. The results are indicated as of the gray scale ratio of each interest protein to GADPH (B,D). Data shown represents the Mean \pm SD of two independent experiments. ${ }^{* *} P<0.01$; ns means no significance.

whereas $\operatorname{sig} B$ was slightly upregulated (Figure $3 B$ ). However, these two genes were markedly repressed by ArgR when bacterial cells were exposed to acidic $\mathrm{pH}$ in the absence of arginine (Figure 3C), but addition of arginine weakened the effect of ArgR on transcription of $\operatorname{arc} A$ and $\operatorname{sig} B$ regardless of $\mathrm{pH}$ conditions (Figures 3E,F). Therefore, L. monocytogenes ArgR appears to be a functional transcriptional regulator that modulates the expression of the arc operon positively and negatively under neutral and acidic $\mathrm{pH}$ conditions, respectively, by employing arginine as a cofactor.

Immuno-blotting was used to determine the relevance of $\operatorname{ArgR}$ to the expression of arginine metabolism operon proteins ArgG and ArcA as well as SigB under neutral and acidic $\mathrm{pH}$ (5.5) conditions. Expression of ArgG in $\Delta \mathrm{ArgR}$ strain was significantly higher under neutral and acidic environments than that of the wild-type strain. (Figures 4A,B). When exogenous arginine was added, expression of ArgG was not detected in the wild-type strain, but expression strongly increased when $\mathrm{ArgR}$ was absent (Figures 4C,D), further indicating that arginine cooperates with ArgR to repress the arginine biosynthetic pathway in Listeria. In addition, $\mathrm{ArgR}$ can regulate the expression of ArcA and SigB in an arginine-dependent and independent manner (Figure 4), which is consistent with the results from transcriptional analysis mentioned above.

\section{Deletion of ArgR Enhances Survival of L. monocytogenes at Lethal Acidic pH}

In order to investigate the contribution of ArgR to the survival of the bacterium at lethal $\mathrm{pH}$ values, acid tolerance experiments were carried out on the mutants in complex medium adjusted to a lethal $\mathrm{pH}$ of 3.5 using $3 \mathrm{M}$ lactic acid. Data show that deletion of $\operatorname{argR}$ exhibited no significant difference in the rate of survival relative to the parent strain at the early time points (30 and 60 min; Figure 5). However, a notable increase in the number of surviving cells was observed for the $\Delta \operatorname{ArgR}$ from minutes 90 onward, as compared to those of the wild-type strain (Figure 5). Conversely, constitutive overexpression of ArgR compromised bacterial survival under the same $\mathrm{pH}$ conditions (Figure 5). It's worth noting that these data are contradictory to findings by Ryan et al. (2009) who reported that L. monocytogenes $\Delta \operatorname{ArgR}$ had defect in acid resistance at both sublethal and lethal $\mathrm{pH}$ levels (Ryan et al., 2009). Nonetheless, based on our findings for ArgR involved in regulations on $\operatorname{arc} A$ and $\operatorname{sig} B$, we speculated that increasing of the acidic survival in the absence of ArgR was most probably due to the activation $\operatorname{arcA}$ and $\operatorname{sig} B$. In addition, consistent with our previous studies (Cheng et al., 2015), survival of $L$. monocytogenes was markedly compromised in the absence of $\operatorname{sig} B$ in the lethal acidic conditions (Figure 5).

\section{DISCUSSION}

The current study demonstrates that $L$. monocytogenes deploys ArgR to control arginine metabolism by negative regulation of arginine metabolism associated genes via binding to the putative ARG box operators as previously described (Fulde et al., 2011; Xiong et al., 2015). Structure modeling and oligomerization analysis indicate that $L$. monocytogenes ArgR has features similar to those of arginine repressors from other bacteria species, in 

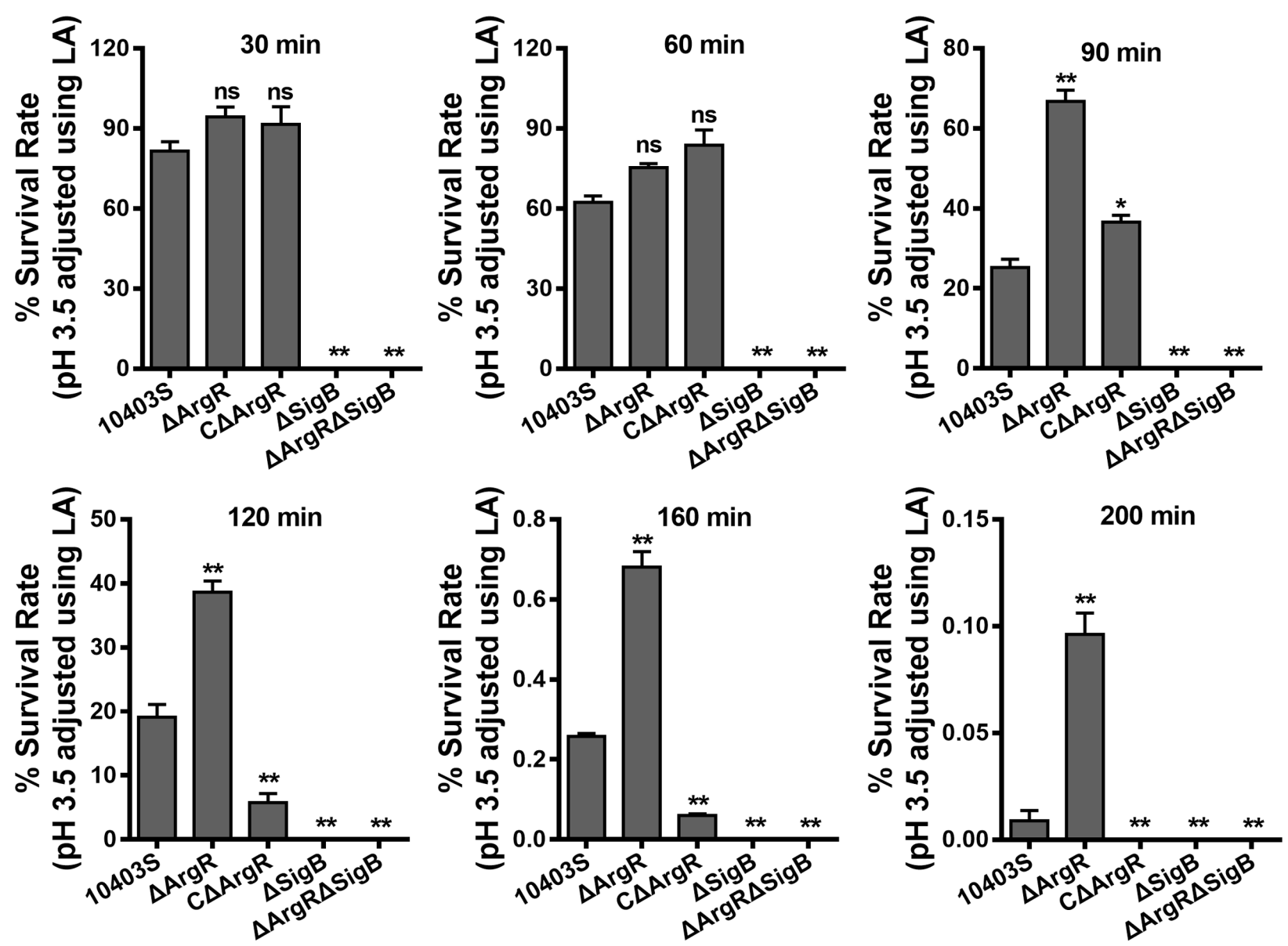

FIGURE 5 | Deletion of ArgR enhances survival of $\boldsymbol{L}$. monocytogenes in the lethal acidic conditions. Overnight-grown $L$. monocytogenes wild-type and mutant strains were harvested, washed and then incubated in $\mathrm{BHI}$ broth (pre-adjusted to $\mathrm{pH} 3.5$ using $3 \mathrm{M}$ lactic acid, LA) at $37^{\circ} \mathrm{C}$. Survivors were enumerated at regular intervals by plating serial dilutions on $\mathrm{BH}$ plate. Data are expressed as Mean $\pm \mathrm{SD}$ of recovery rate for each strain. ${ }^{*} P<0.05$; $* * P<0.01$; ns means no significance.

particular with ArgR from B. subtilis (Dennis et al., 2002; Garnett et al., 2007). The N-terminal domain of ArgR is the DBD, whereas the C-terminal domain required for oligomerization and arginine binding (Sunnerhagen et al., 1997; Ni et al., 1999; Garnett et al., 2008). The ArgR protomers can form trimers and hexamers that are in equilibrium and their oligomerization state is manipulated by the presence of arginine corepressor that is bound in the space between ArgR trimers and link each pair of opposite trimers via their guanidinium groups, thereby providing additional stability as hexamer (Ni et al., 1999; Cherney et al., 2009).

As is the case in other bacteria species (Larsen et al., 2004; Nicoloff et al., 2004), we found that ArgR in L. monocytogenes also acts as a negative regulator of the arginine biosynthetic pathway by repression of $\operatorname{argCJBD}$ and $\operatorname{argGH}$ in the absence of ArgR, and such a regulatory effect was augmented under acidic conditions or in the presence of arginine. Generally, ArgR has been demonstrated to act as a positive regulator of $\operatorname{arc} A B C$ operon expression in many bacteria species, which is essential for acid resistance (Griswold et al., 2004; Fulde et al., 2011; Xiong et al., 2015). ArcA and sigB were repressed by ArgR in the absence of extracellular arginine, while such effects were not seen when extracellular arginine was added. Notably, deletion of ArgR markedly enhanced the capacity of L. monocytogenes to survive in the lethal acid environments. However, Ryan et al. (2009) have previously noted that L. monocytogenes $\triangle \mathrm{ArgR}$ demonstrated a great defect in acid resistance at both sublethal and lethal $\mathrm{pH}$ levels. It's worth noting that we used the same acidic conditions (media adjusted to $\mathrm{pH} 3.5$ using $3 \mathrm{M}$ lactic acid) and bacterial growth status as reported by Ryan et al. (2009); however, these authors used L. monocytogenes LO28, a serotype $1 / 2$ c strain (Ryan et al., 2009). We here speculate that the capacity of $L$. monocytogenes to survive in acidic environments was attributable to the activation of $\operatorname{arc} A$ and $\operatorname{sig} B$ in the absence of $\operatorname{ArgR}$. It is well known that in a number of bacterial species, catabolism of arginine via the ADI pathway has been demonstrated to play a critical role in an enhanced capacity to survive under acidic extracellular conditions (Xiong et al., 2014; $\mathrm{Xu}$ et al., 2016), and L. monocytogenes is likely no exception (Ryan et al., 2009; Cheng et al., 2013b). Besides, the alternative factor, SigB has been widely studied as it plays a key role in 


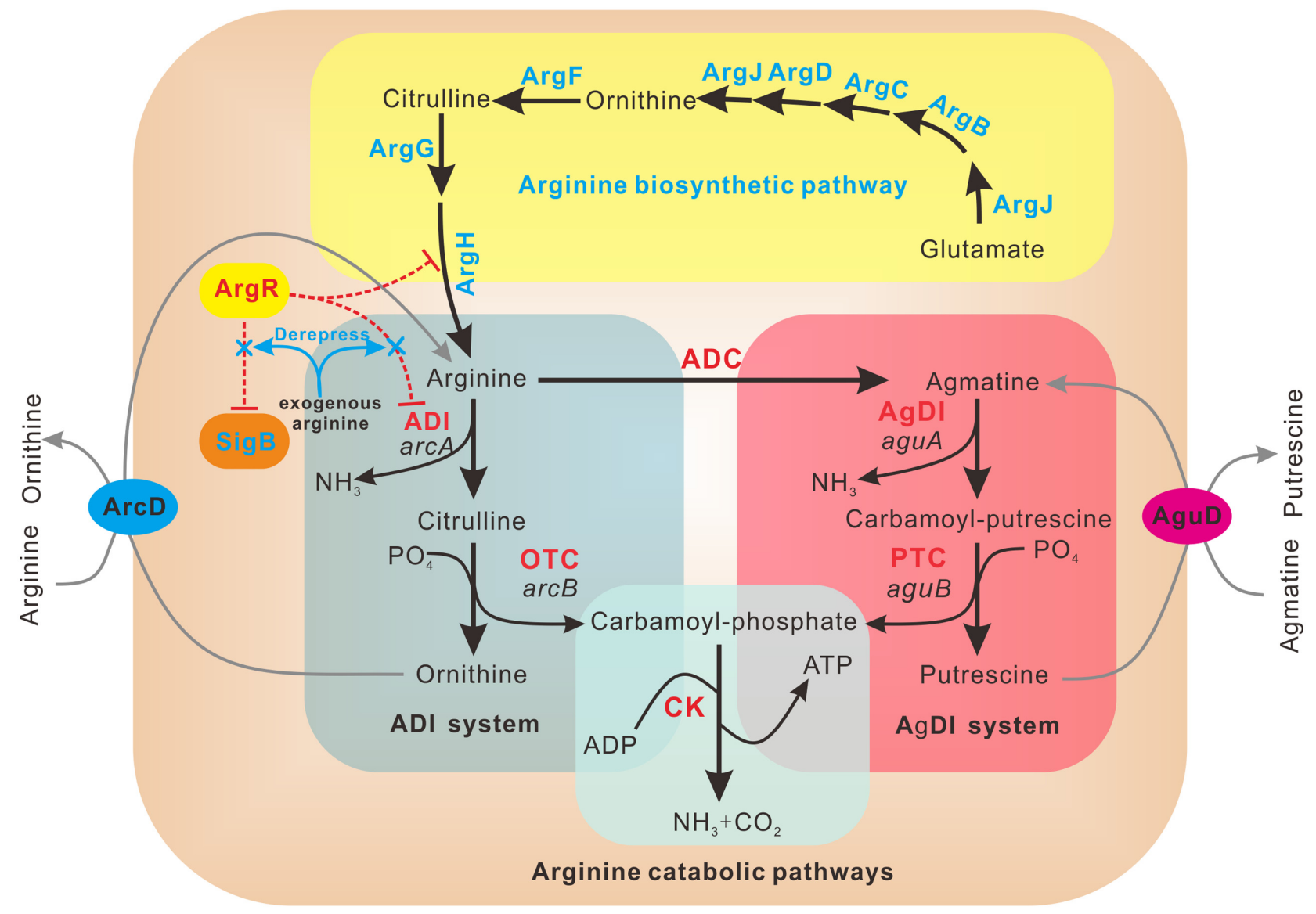

FIGURE 6 | Schematic representation of regulatory mechanism employed by ArgR in L. monocytogenes. L. monocytogenes ArgR plays a classical role of ArgR/AhrC family in feedback inhibitory of arginine biosynthetic pathway (highlighted in yellow) using arginine as a cofactor. ArgR unexpectedly represses the transcription and expression of arcA and sigB in the absence of exogenous arginine, preventing arginine degradation via the ADI and AgDI pathways (highlighted in light cyan and light red, respectively). Addition of arginine leads to derepression of $\operatorname{arc} A$ and sigB, allowing utilization of arginine as a nitrogen and energy source via the arginine degradation pathway.

L. monocytogenes survival under multiple environmental stress conditions, including elevated osmolarity, low $\mathrm{pH}$ and oxidativestresses (Kim et al., 2004; Gahan and Hill, 2005).

This is the first study showing that a single ArgR regulator can have opposite regulatory effects on the ADI pathway in an arginine-independent and dependent manner under neutral and acidic conditions, respectively. However, the underlying molecular mechanisms are still unknown and warrant further study. In general, ArgR-type proteins act as a positive regulator of the ADI system and a negative regulator of the arginine biosynthetic pathway. However, there are two circumstances for unconventional ArgR regulatory mechanisms. One exists in bacteria that encode two ArgR homologs. For instance, the expression of arginine metabolism in Lactococcus lactis is controlled by the two homologous transcriptional regulators ArgR and AhrC. Specifically, ArgR binds to the promoter regions of both the arginine biosynthetic and catabolic operons in an arginine-independent manner. With both regulators present, addition of arginine leads to increased binding of ArgR-AhrC to the biosynthetic $\operatorname{argC}$ promoter but also to diminished binding to the catabolic $\operatorname{arcA}$ promoter (Larsen et al., 2005). The other circumstance is for the bacteria that contain one single ArgR homologous but two arc operons. Xiong et al. (2015) has demonstrated that arginine catabolism in Laribacter hongkongensis is finely regulated by manipulating the transcription of two arc operons. L. hongkongensis ArgR exhibited an opposite effect on transcription and expression of these two arc operons. In the presence of arginine, deletion of $\arg R$ partially compromised the repressive effects that arginine had on $\operatorname{arcA} 1$ expression; while it dramatically decreased the transcriptional levels of $\operatorname{arcA} 2$ (Xiong et al., 2015).

Since L. monocytogenes encodes a single ArgR homolog and one arc operon, we speculate that ArgR maintains its functions as a unique transcriptional regulator with dual regulatory effects on ADI pathway and SigB under different environmental stresses. Based on the results presented here, we propose a model depicting the mechanisms of ArgR in arginine-meditated transcriptional regulation in L. monocytogenes (Figure 6). In the absence of arginine, ArgR shows higher affinity for arc operon promoter, and relatively lower affinity for arg operons 
compared to that in the presence of arginine, consequently preventing arginine degradation via the ADI pathway and repressing arginine biosynthesis to a low extent. The addition of arginine shifts ArgR from the $\operatorname{arcA}$ promoter to the ARG box operators in the $\arg$ operons, which enhances repression of the arginine biosynthetic genes. Accordingly, the arginine catabolic arc operon is now derepressed, allowing catabolism of arginine as a nitrogen and energy source through the ADI arginine degradation pathway. Therefore, L. monocytogenes ArgR appears to have unusual roles in repression of arginine biosynthetic operon in an arginine-independent manner, and activation of catabolism by anti-repression in an arginine-dependent way.

\section{AUTHOR CONTRIBUTIONS}

CC, WF, and HS conceived the study. CC, JS, XH, ZD, HW, LJ, and TM carried out experiments. CC, YY, ZC, and JY analyzed data. CC, WF, and HS drafted the manuscript and all the authors contributed to preparing the final version of the manuscript. All authors read and approved the final manuscript.

\section{FUNDING}

This work was supported by National Natural Science Foundation of China (Nos. 31470179, 31502083, and 31402215),

\section{REFERENCES}

Arnold, K., Bordoli, L., Kopp, J., and Schwede, T. (2006). The SWISSMODEL workspace: a web-based environment for protein structure homology modelling. Bioinformatics 22, 195-201. doi: 10.1093/bioinformatics/bti770

Bordoli, L., Kiefer, F., Arnold, K., Benkert, P., Battey, J., and Schwede, T. (2009). Protein structure homology modeling using SWISS-MODEL workspace. Nat. Protoc. 4, 1-13. doi: 10.1038/nprot.2008.197

Bordoli, L., and Schwede, T. (2012). Automated protein structure modeling with SWISS-MODEL workspace and the protein model portal. Methods Mol. Biol. 857, 107-136. doi: 10.1007/978-1-61779-588-6_5

Boylan, S. A., Redfield, A. R., Brody, M. S., and Price, C. W. (1993). Stress-induced activation of the sigma B transcription factor of Bacillus subtilis. J. Bacteriol. 175, 7931-7937. doi: 10.1128/jb.175.24.7931-7937.1993

Caldara, M., Charlier, D., and Cunin, R. (2006). The arginine regulon of Escherichia coli: whole-system transcriptome analysis discovers new genes and provides an integrated view of arginine regulation. Microbiology 152, 3343-3354. doi: 10.1099/mic.0.29088-0

Camilli, A., Tilney, L. G., and Portnoy, D. A. (1993). Dual roles of plcA in Listeria monocytogenes pathogenesis. Mol. Microbiol. 8, 143-157. doi: 10.1111/j.13652958.1993.tb01211.x

Chen, J., Cheng, C., Xia, Y., Zhao, H., Fang, C., Shan, Y., et al. (2011). Lmo0036, an ornithine and putrescine carbamoyltransferase in Listeria monocytogenes, participates in arginine deiminase and agmatine deiminase pathways and mediates acid tolerance. Microbiology 157(Pt 11), 3150-3161. doi: 10.1099/mic. 0.049619-0

Chen, S. H., Merican, A. F., and Sherratt, D. J. (1997). DNA binding of Escherichia coli arginine repressor mutants altered in oligomeric state. Mol. Microbiol. 24, 1143-1156. doi: 10.1046/j.1365-2958.1997.4301791.x

Cheng, C., Chen, J., Fang, C., Xia, Y., Shan, Y., Liu, Y., et al. (2013a). Listeria monocytogenes aguA1, but not aguA2, encodes a functional agmatine deiminase: biochemical characterization of its catalytic properties and roles in acid tolerance. J. Biol. Chem. 288, 26606-26615. doi: 10.1074/jbc.M113.477380
Zhejiang Provincial Natural Science Foundation (Nos. LY17C180001, LY15C010003, and LQ14C010007) and ZAFU talents starting program (Nos. 2014FR073). The funders had no role in design of the study or analysis and interpretation of the data.

\section{ACKNOWLEDGMENTS}

We specially thank Dr. Martin Wiedmann at Cornell University and Dr. Nancy Freitag at University of Illinois at Chicago for kindly providing the shuttle plasmids pKSV7 and pIMK2, respectively.

\section{SUPPLEMENTARY MATERIAL}

The Supplementary Material for this article can be found online at: http://journal.frontiersin.org/article/10.3389/fmicb. 2017.00145/full\#supplementary-material

FIGURE S1 | The arginine biosynthetic pathway and organization of the gene cluster in L. monocytogenes. (A) The arginine biosynthetic pathway. Arginine is synthesized from glutamate in eight steps catalyzed by a series of enzymes encoded by argABCDEFGH. Five steps involving $N$-acetylated intermediates lead to ornithine, and three additional steps are required to convert ornithine into arginine. The synthesis of all enzymes is subject to repression by arginine, mediated by the repressor ArgR. (B) Genetic organization of the arginine biosynthesis pathway gene cluster in L. monocytogenes strain $10403 S$.

Cheng, C., Chen, J., Shan, Y., Fang, C., Liu, Y., Xia, Y., et al. (2013b). Listeria monocytogenes ArcA contributes to acid tolerance. J. Med. Microbiol. 62(Pt 6), 813-821. doi: 10.1099/jmm.0.055145-0

Cheng, C., Yang, Y., Dong, Z., Wang, X., Fang, C., Yang, M., et al. (2015). Listeria monocytogenes varies among strains to maintain intracellular $\mathrm{pH}$ homeostasis under stresses by different acids as analyzed by a high-throughput microplatebased fluorometry. Front. Microbiol. 6:15. doi: 10.3389/fmicb.2015.00015

Cherney, L. T., Cherney, M. M., Garen, C. R., and James, M. N. (2009). The structure of the arginine repressor from Mycobacterium tuberculosis bound with its DNA operator and Co-repressor, L-arginine. J. Mol. Biol. 388, 85-97. doi: 10.1016/j.jmb.2009.02.053

Choi, Y., Choi, J., Groisman, E. A., Kang, D. H., Shin, D., and Ryu, S. (2012). Expression of STM4467-encoded arginine deiminase controlled by the STM4463 regulator contributes to Salmonella enterica serovar Typhimurium virulence. Infect. Immun. 80, 4291-4297. doi: 10.1128/IAI. 00880-12

Cossart, P. (2011). Illuminating the landscape of host-pathogen interactions with the bacterium Listeria monocytogenes. Proc. Natl. Acad. Sci. U.S.A. 108, 19484-19491. doi: 10.1073/pnas.1112371108

Cotter, P. D., and Hill, C. (2003). Surviving the acid test: responses of gram-positive bacteria to low pH. Microbiol. Mol. Biol. Rev. 67, 429-453. doi: 10.1128/MMBR. 67.3.429-453.2003

Cunin, R., Glansdorff, N., Pierard, A., and Stalon, V. (1986). Biosynthesis and metabolism of arginine in bacteria. Microbiol. Rev. 50, 314-352.

Dennis, C. A., Glykos, N. M., Parsons, M. R., and Phillips, S. E. V. (2002). The structure of AhrC, the arginine repressor/activator protein from Bacillus subtilis. Acta Crystallogr. D-Biol. Crystallogr. 58, 421-430. doi: 10.1107/ S0907444901021692

Dong, Y., Chen, Y. Y., and Burne, R. A. (2004). Control of expression of the arginine deiminase operon of Streptococcus gordonii by CcpA and Flp. J. Bacteriol. 186, 2511-2514. doi: 10.1128/JB.186.8.2511-2514.2004

Edgar, R. C. (2004). MUSCLE: multiple sequence alignment with high accuracy and high throughput. Nucleic Acids Res. 32, 1792-1797. doi: 10.1093/nar/gkh340 
Ferreira, A., O'Byrne, C. P., and Boor, K. J. (2001). Role of sigma(B) in heat, ethanol, acid, and oxidative stress resistance and during carbon starvation in Listeria monocytogenes. Appl. Environ. Microbiol. 67, 4454-4457. doi: 10.1128/AEM.67. 10.4454-4457.2001

Fulde, M., Willenborg, J., de Greeff, A., Benga, L., Smith, H. E., ValentinWeigand, P., et al. (2011). ArgR is an essential local transcriptional regulator of the arcABC operon in Streptococcus suis and is crucial for biological fitness in an acidic environment. Microbiology 157(Pt 2), 572-582. doi: 10.1099/mic.0. 043067-0

Gahan, C. G., and Hill, C. (2005). Gastrointestinal phase of Listeria monocytogenes infection. J. Appl. Microbiol. 98, 1345-1353. doi: 10.1111/j.1365-2672.2005. 02559.x

Garnett, J. A., Baumberg, S., Stockley, P. G., and Phillips, S. E. (2007). A highresolution structure of the DNA-binding domain of AhrC, the arginine repressor/activator protein from Bacillus subtilis. Acta Crystallogr. Sect. F Struct. Biol. Cryst. Commun. 63(Pt 11), 914-917. doi: 10.1107/S1744309107048166

Garnett, J. A., Marincs, F., Baumberg, S., Stockley, P. G., and Phillips, S. E. (2008). Structure and function of the arginine repressor-operator complex from Bacillus subtilis. J. Mol. Biol. 379, 284-298. doi: 10.1016/j.jmb.2008.03.007

Glaser, P., Frangeul, L., Buchrieser, C., Rusniok, C., Amend, A., Baquero, F., et al. (2001). Comparative genomics of Listeria species. Science 294, 849-852. doi: 10.1126/science.1063447

Gray, M. J., Freitag, N. E., and Boor, K. J. (2006). How the bacterial pathogen Listeria monocytogenes mediates the switch from environmental Dr. Jekyll to pathogenic Mr. Hyde. Infect. Immun. 74, 2505-2512. doi: 10.1128/IAI.74.5. 2505-2512.2006

Griswold, A., Chen, Y. Y., Snyder, J. A., and Burne, R. A. (2004). Characterization of the arginine deiminase operon of Streptococcus rattus FA-1. Appl. Environ. Microbiol. 70, 1321-1327. doi: 10.1128/AEM.70.3.1321-1327.2004

Gruening, P., Fulde, M., Valentin-Weigand, P., and Goethe, R. (2006). Structure, regulation, and putative function of the arginine deiminase system of Streptococcus suis. J. Bacteriol. 188, 361-369. doi: 10.1128/JB.188.2.361-369. 2006

Kim, H., Boor, K. J., and Marquis, H. (2004). Listeria monocytogenes sigmaB contributes to invasion of human intestinal epithelial cells. Infect. Immun. 72, 7374-7378. doi: 10.1128/IAI.72.12.7374-7378.2004

Kloosterman, T. G., and Kuipers, O. P. (2011). Regulation of arginine acquisition and virulence gene expression in the human pathogen Streptococcus pneumoniae by transcription regulators ArgR1 and AhrC. J. Biol. Chem. 286, 44594-44605. doi: 10.1074/jbc.M111.295832

Larsen, R., Buist, G., Kuipers, O. P., and Kok, J. (2004). ArgR and AhrC are both required for regulation of arginine metabolism in Lactococcus lactis. J. Bacteriol. 186, 1147-1157. doi: 10.1128/JB.186.4.1147-1157.2004

Larsen, R., Kok, J., and Kuipers, O. P. (2005). Interaction between ArgR and AhrC controls regulation of arginine metabolism in Lactococcus lactis. J. Biol. Chem. 280, 19319-19330. doi: 10.1074/jbc.M413983200

Lebreton, A., Stavru, F., and Cossart, P. (2015). Organelle targeting during bacterial infection: insights from Listeria. Trends Cell Biol. 25, 330-338. doi: 10.1016/j. tcb.2015.01.003

Livak, K. J., and Schmittgen, T. D. (2001). Analysis of relative gene expression data using real-time quantitative PCR and the $2^{\Delta \Delta C T}$ method. Methods 25, 402-408. doi: 10.1006/meth.2001.1262

Lu, C. D. (2006). Pathways and regulation of bacterial arginine metabolism and perspectives for obtaining arginine overproducing strains. Appl. Microbiol. Biotechnol. 70, 261-272. doi: 10.1007/s00253-005-0308-z

Lucas, P. M., Blancato, V. S., Claisse, O., Magni, C., Lolkema, J. S., and LonvaudFunel, A. (2007). Agmatine deiminase pathway genes in Lactobacillus brevis are linked to the tyrosine decarboxylation operon in a putative acid resistance locus. Microbiology 153(Pt 7), 2221-2230. doi: 10.1099/mic.0.2007/006320-0

Maas, W. K. (1994). The arginine repressor of Escherichia coli. Microbiol. Rev. 58, 631-640.

Maghnouj, A., de Sousa Cabral, T. F., Stalon, V., and Vander Wauven, C. (1998). The arcABDC gene cluster, encoding the arginine deiminase pathway of Bacillus licheniformis, and its activation by the arginine repressor argR. J. Bacteriol. 180, 6468-6475.

Makarova, K. S., Mironov, A. A., and Gelfand, M. S. (2001). Conservation of the binding site for the arginine repressor in all bacterial lineages. Genome Biol. 2:RESEARCH0013. doi: 10.1186/gb-2001-2-9-research0033
Marquis, R. E., Bender, G. R., Murray, D. R., and Wong, A. (1987). Arginine deiminase system and bacterial adaptation to acid environments. Appl. Environ. Microbiol. 53, 198-200.

Miller, C. M., Baumberg, S., and Stockley, P. G. (1997). Operator interactions by the Bacillus subtilis arginine repressor/activator, AhrC: novel positioning and DNA-mediated assembly of a transcriptional activator at catabolic sites. Mol. Microbiol. 26, 37-48.

Monk, I. R., Gahan, C. G., and Hill, C. (2008). Tools for functional postgenomic analysis of Listeria monocytogenes. Appl. Environ. Microbiol. 74, 3921-3934. doi: 10.1128/AEM.00314-08

Munch, R., Hiller, K., Grote, A., Scheer, M., Klein, J., Schobert, M., et al. (2005). Virtual Footprint and PRODORIC: an integrative framework for regulon prediction in prokaryotes. Bioinformatics 21, 4187-4189. doi: 10.1093/ bioinformatics/bti635

Ni, J., Sakanyan, V., Charlier, D., Glansdorff, N., and Van Duyne, G. D. (1999). Structure of the arginine repressor from Bacillus stearothermophilus. Nat. Struct. Biol. 6, 427-432. doi: 10.1038/8229

Nicoloff, H., Arsene-Ploetze, F., Malandain, C., Kleerebezem, M., and Bringel, F. (2004). Two arginine repressors regulate arginine biosynthesis in Lactobacillus plantarum. J. Bacteriol. 186, 6059-6069. doi: 10.1128/JB.186.18.6059-6069.2004

Palmer, M. E., Chaturongakul, S., Wiedmann, M., and Boor, K. J. (2011). The Listeria monocytogenes sigmaB regulon and its virulence-associated functions are inhibited by a small molecule. MBio 2:e241-11. doi: 10.1128/mBio.00241-11

Perez-Redondo, R., Rodriguez-Garcia, A., Botas, A., Santamarta, I., Martin, J. F., and Liras, P. (2012). ArgR of Streptomyces coelicolor is a versatile regulator. PLoS ONE 7:e32697. doi: 10.1371/journal.pone.0032697

Rodriguez-Garcia, A., Ludovice, M., Martin, J. F., and Liras, P. (1997). Arginine boxes and the argR gene in Streptomyces clavuligerus: evidence for a clear regulation of the arginine pathway. Mol. Microbiol. 25, 219-228. doi: 10.1046/j. 1365-2958.1997.4511815.x

Ryan, S., Begley, M., Gahan, C. G., and Hill, C. (2009). Molecular characterization of the arginine deiminase system in Listeria monocytogenes: regulation and role in acid tolerance. Environ. Microbiol. 11, 432-445. doi: 10.1111/j.1462-2920. 2008.01782.x

Sunnerhagen, M., Nilges, M., Otting, G., and Carey, J. (1997). Solution structure of the DNA-binding domain and model for the complex of multifunctional hexameric arginine repressor with DNA. Nat. Struct. Biol. 4, 819-826. doi: 10.1038/nsb1097-819

Xia, Y., Xin, Y., Li, X., and Fang, W. (2016). To modulate survival under secondary stress sonditions, Listeria monocytogenes $10403 \mathrm{~S}$ employs RsbX to downregulate sigmaB activity in the poststress recovery stage or stationary phase. Appl. Environ. Microbiol. 82, 1126-1135. doi: 10.1128/AEM.03218-15

Xiong, L., Teng, J. L., Watt, R. M., Kan, B., Lau, S. K., and Woo, P. C. (2014). Arginine deiminase pathway is far more important than urease for acid resistance and intracellular survival in Laribacter hongkongensis: a possible result of arc gene cassette duplication. BMC Microbiol. 14:42. doi: 10.1186/14712180-14-42

Xiong, L., Teng, J. L., Watt, R. M., Liu, C., Lau, S. K., and Woo, P. C. (2015). Molecular characterization of arginine deiminase pathway in Laribacter hongkongensis and unique regulation of arginine catabolism and anabolism by multiple environmental stresses. Environ. Microbiol. 17, 4469-4483. doi: $10.1111 / 1462-2920.12897$

Xu, B., Yang, X., Zhang, P., Ma, Z., Lin, H., and Fan, H. (2016). The arginine deiminase system facilitates environmental adaptability of Streptococcus equi ssp. zooepidemicus through pH adjustment. Res. Microbiol. 167, 403-412. doi: 10.1016/j.resmic.2016.03.005

Conflict of Interest Statement: The authors declare that the research was conducted in the absence of any commercial or financial relationships that could be construed as a potential conflict of interest.

Copyright (c) 2017 Cheng, Dong, Han, Sun, Wang, Jiang, Yang, Ma, Chen, Yu, Fang and Song. This is an open-access article distributed under the terms of the Creative Commons Attribution License (CC BY). The use, distribution or reproduction in other forums is permitted, provided the original author(s) or licensor are credited and that the original publication in this journal is cited, in accordance with accepted academic practice. No use, distribution or reproduction is permitted which does not comply with these terms. 